\title{
Random Benzotrithiophene-Based Donor-Acceptor Copolymers for Efficient Organic Photovoltaic Devices
}

\author{
Christian B. Nielsen, ${ }^{* a}$ Raja Shahid Ashraf, ${ }^{a}$ Bob C. Schroeder, ${ }^{a}$ Pasquale D'Angelo,${ }^{b}$ Scott E. Watkins, ${ }^{c}$ \\ Kigook Song, ${ }^{d}$ Thomas D. Anthopoulos, ${ }^{b}$ and Iain McCulloch ${ }^{a}$
}

${ }_{5}$ Received (in $\left.X X X, X X X\right) X$ th $X X X X X X X X X 20 X X$, Accepted Xth $X X X X X X X X X 20 X X$ DOI: $10.1039 / \mathrm{b000000x}$

\begin{abstract}
A series of benzotrithiophene-containing random terpolymers for polymer solar cells is reported. Through variations of the two other components in the terpolymers, the absorption ${ }_{10}$ profile and the frontier energy levels are optimized and maximum power conversion efficiencies are nearly doubled $(5.14 \%)$ relative to the parent alternating copolymer.
\end{abstract}

Research in organic photovoltaics (OPV) has been attracting much attention over recent years and the development of new 15 light-harvesting donor materials for bulk-heterojunction (BHJ) solar cells with fullerene-based electron acceptors has been progressing rapidly with solar cell efficiencies fast approaching $10 \% .^{1,2}$ Judicious adjustment of frontier energy levels is an integral part of the process when designing new donor materials 20 for OPV applications. ${ }^{3,4}$ This is, firstly, to ensure efficient charge transfer from the excited donor material to the acceptor material and, secondly, to optimize the inherent trade-off associated with pursuing both a high open-circuit voltage and a favourable spectral overlap with the solar radiation.

${ }_{25}$ Several strategies to broaden the absorption spectra of the light-harvesting materials and thus better match the solar spectrum can be envisioned. By combining two solar cells in series to create a tandem solar cell, one can utilize two complementary donor materials with different absorption bands 30 and effectively harvest a larger proportion of the solar radiation. 5 Alternatively, the incorporation of several different chromophores into one polymeric donor material by means of a random copolymerization approach has also been proven to effectively broaden the absorption spectrum. ${ }^{7}$ The latter, single 35 component strategy has recently been applied in OPV devices with some success although clear evidence of significant improvements over the parent systems is limited. ${ }^{8-10}$

We have previously reported on the synthesis of benzo[1,2$\mathrm{b}: 3,4-\mathrm{b} ' 5,6$-d'']trithiophene (BTT) and on BTT-BT $(\mathrm{BT}=$ 40 benzothiadiazole) and BTT-DPP (DPP = diketopyrrolopyrrole) alternating donor-acceptor type copolymers for photovoltaic applications with moderate efficiencies. ${ }^{11-13}$ Here we describe a highly successful approach to improving the OPV efficiency significantly by randomly copolymerizing BTT and DPP with a

45 third monomer as illustrated in Scheme 1. By varying the nature and the content of the third monomer we are able to fine tune the frontier energy levels as well as significantly optimise the optical absorption spectrum. Additionally, both the solubility and phase separation can also be further enhanced. As a direct result hereof, 50 we are now able to demonstrate solar cells with power conversion efficiencies as high as $5.14 \%$ with these random terpolymers, compared to $2.68 \%$ for the alternating copolymer analogue.

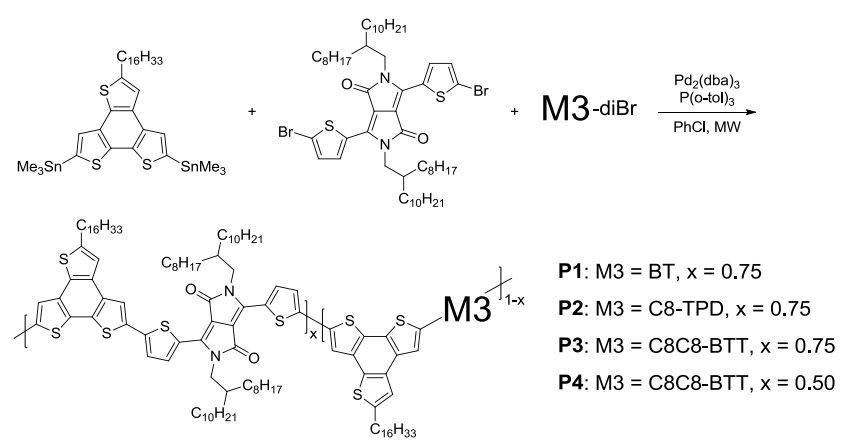

Scheme 1 Synthesis of random BTT-containing copolymers $(\mathrm{BT}=2,1,3$ 55 benzothiadiazole, C8-TPD $=N$-octylthienopyrrolodione, $\mathrm{C} 8 \mathrm{C} 8=1$ octylnonyl).

As illustrated in Scheme 1, three-component microwaveassisted Stille polycondensations were carried out by reacting one equivalent of distannylated C16-BTT with one equivalent of a ${ }_{60}$ dibrominated mixture of DPP and a third comonomer M3. First, to incorporate weaker acceptor units than DPP, P1 and P2 were synthesized by using BT and C8-TPD respectively as M3 with a DPP/M3 ratio of 3:1 in both cases. Secondly, to effectively lower the DPP content, random terpolymers P3 (DPP/M3 ratio 3:1) and ${ }_{65} \mathbf{P 4}$ (DPP/M3 ratio 1:1) were obtained with C8C8-BTT (using a branched alkyl chain for increased solubility) as the third comonomer. Additionally, the alternating BTT-DPP copolymer was prepared from equimolar amounts of distannylated C16-BTT and dibrominated DPP using identical reaction conditions. 
Table 1 Molecular Weights and Thermal Stability of the Polymers

$\begin{array}{ccccc}\text { Polymer } & M_{\mathrm{n}}{ }^{a}(\mathrm{~kg} / \mathrm{mol}) & M_{\mathrm{w}}{ }^{b}(\mathrm{~kg} / \mathrm{mol}) & \mathrm{PDI}^{c} & T_{\mathrm{d}}{ }^{d}\left({ }^{\circ} \mathrm{C}\right) \\ \text { BTT-DPP } & 185 & 580 & 3.1 & 391 \\ \text { P1 } & 130 & 280 & 2.1 & 415 \\ \text { P2 } & 90 & 130 & 1.4 & 416 \\ \text { P3 } & 190 & 520 & 2.7 & 420 \\ \text { P4 } & 165 & 430 & 2.6 & 426\end{array}$

${ }^{a}$ Number-average molecular weight. ${ }^{b}$ Weight-average molecular weight. ${ }^{c} M_{\mathrm{w}} / M_{\mathrm{n}} \cdot{ }^{d}$ Decomposition temperature (5\% weight loss) determined by thermal gravimetric analysis under nitrogen.

5 All polymers were obtained as thermally stable, black fibrous materials in good yields and with high molecular weights as summarized in Table 1. Differential scanning calorimetry (DSC) (Figure S2 in the Supporting Information) did not show any thermal transitions for any of the polymers. Interestingly, whereas 10 all four random polymerizations (synthesis of P1-P4) proceeded easily to give very high degrees of polymerization even in the crude reaction mixture, the alternating BTT-DPP polymer was repeatedly obtained with a much lower crude molecular weight. It required further fractionation by means of preparative GPC to 15 afford a comparably high molecular weight sample of this polymer. We ascribe this difference to the reduced solubility of the alternating copolymer compared to the random terpolymers.
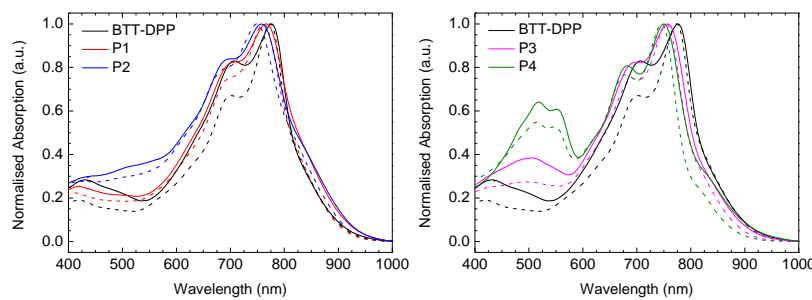

Figure 1 Normalized optical absorption spectra of BTT-DPP, P1 and P2

$20 \quad$ (left) and BTT-DPP, $\mathbf{P 3}$ and $\mathbf{P 4}$ (right) in dilute CB solution (dashed

lines) and as thin films (full lines) spin-cast from CB solution $(5 \mathrm{mg} / \mathrm{ml})$.

Optical absorption spectra of BTT-DPP, P1, and P2 in chlorobenzene $(\mathrm{CB})$ solution and as thin films cast from $\mathrm{CB}$ solution are displayed in Figure 1 (left) and summarized in Table 25 2. Compared to BTT-DPP, a slight blue-shift in absorption maximum is observed both in solution and in the solid state when incorporating BT into the polymer (P1). A further blue-shift is observed with the weaker acceptor unit, TPD, (P2) as well as a slight broadening of the absorption band. The optical band gaps 30 as determined from the onset of absorption in the solid state are found to be almost identical for the three polymers (1.34-1.36 eV). Upon comparison of the optical absorption of BTT-DPP, P3, and P4 (Figure 1 (right) and Table 2), a similar trend is observed with a gradual blue-shift in absorption maximum when 35 going from BTT-DPP to P3 and from P3 to P4 caused by the decrease in DPP-content. Moreover, the increase in BTT-content results in the appearance of a new absorption band around 500 $\mathrm{nm}$, which is most likely from the $\pi-\pi^{*}$ transition of oligomeric BTT chromophores within the polymer chain. Again, the optical 40 band gaps are found to be practically identical for the series of polymers $(1.36-1.37 \mathrm{eV})$.
Table 2 Optical Properties and Frontier Energy Levels of the Polymers

$\begin{array}{cccccc}\text { Polymer } & \lambda_{\max }{ }^{a}(\mathrm{~nm}) & \lambda_{\max }{ }^{b}(\mathrm{~nm}) & E_{\mathrm{g}}{ }^{c} & \mathrm{HOMO}^{d}(\mathrm{eV}) & \mathrm{LUMO}^{e}(\mathrm{eV}) \\ \text { BTT-DPP } & 774 & 776 & 1.36 & -5.2 & -3.8 \\ \text { P1 } & 761 & 767 & 1.36 & -5.2 & -3.8 \\ \text { P2 } & 748 & 758 & 1.34 & -5.3 & -3.9 \\ \text { P3 } & 755 & 757 & 1.37 & -5.2 & -3.8 \\ \text { P4 } & 746 & 750 & 1.37 & -5.1 & -3.7\end{array}$

${ }^{a}$ Dilute CB solution. ${ }^{b}$ Thin film spin-cast from CB solution $(5 \mathrm{mg} / \mathrm{ml}$, $1000 \mathrm{rpm}) .{ }^{c}$ Optical band gap determined from the onset of absorption in 45 the solid state. ${ }^{d}$ HOMO energy level measured by photoelectron spectroscopy in air. ${ }^{e}$ LUMO energy level estimated from the HOMO level and the optical band gap.

The highest occupied molecular orbital (HOMO) energy levels were determined by photoelectron spectroscopy in air (Table 2). 50 HOMO energy levels of $-5.2 \mathrm{eV}$ are found for both BTT-DPP and P1. Substituting BT with TPD has previously been shown to lower the HOMO level slightly and we observe this trend here as well with $\mathbf{P 2}$ having a HOMO level of $-5.3 \mathrm{eV} .{ }^{14}$ Polymers P3 and $\mathbf{P 4}$ are expected to be more electron-rich than BTT-DPP due 55 to the increased BTT-content and this is indeed reflected in a slightly higher HOMO value of $-5.1 \mathrm{eV}$ for P4. Lowest unoccupied molecular orbital (LUMO) energy levels can be estimated from the measured HOMO levels and the optical band gaps (Table 2). For all polymers the LUMO level is expected to 60 be mainly governed by the strongly electron-accepting DPP unit. LUMOs are found to be in the range -3.7--3.9 eV, with the highest value of $-3.7 \mathrm{eV}$ observed for $\mathbf{P} \mathbf{4}$, which contains the least amount of DPP. P2, on the other hand, is estimated to have a slightly deeper LUMO $(-3.9 \mathrm{eV})$ than the rest of the polymers.

65 Table 3 Photovoltaic Properties of the Polymers

\begin{tabular}{ccccc}
\hline Polymer & $J_{\mathrm{SC}}\left(\mathrm{mA} / \mathrm{cm}^{2}\right)$ & $V_{\mathrm{OC}}(\mathrm{V})$ & FF & PCE $(\%)$ \\
BTT-DPP & 6.30 & 0.71 & 0.60 & 2.68 \\
P1 & 10.95 & 0.68 & 0.69 & 5.14 \\
P2 & 8.87 & 0.72 & 0.66 & 4.23 \\
P3 & 11.10 & 0.68 & 0.61 & 4.58 \\
P4 & 12.07 & 0.66 & 0.53 & 4.28
\end{tabular}

${ }^{a}$ Devices with a polymer: $\mathrm{PC}_{71} \mathrm{BM}$ blend ratio of $1: 2$ processed from chloroform:o-dichlorobenzene (4:1). Device configuration ITO/PEDOT:PSS/polymer:PC ${ }_{71} \mathrm{BM} / \mathrm{LiF} / \mathrm{Al}$; tested under simulated 100 $\mathrm{mW} / \mathrm{cm}^{2} \mathrm{AM} 1.5 \mathrm{G}$ illumination.
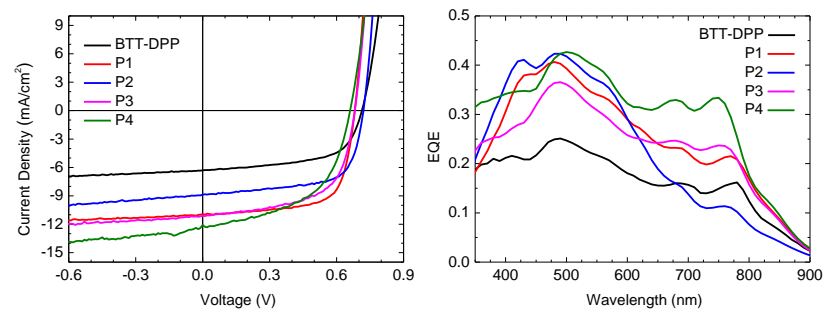

Figure $2 \mathrm{~J}-\mathrm{V}$ characteristics (left) and external quantum efficiencies (right) of the OPV devices.

In solar cell devices (Figure 2 and Table 3), the alternating BTT-DPP polymer afforded an open-circuit voltage $\left(V_{\mathrm{OC}}\right)$ of $750.71 \mathrm{~V}$ and a fill factor (FF) of 0.60 ; values which are comparable to some of the best performing DPP-polymers. The short-circuit current $\left(J_{\mathrm{SC}}\right)$, on the other hand, was found to be rather low $(6.30$ 
$\mathrm{mA} / \mathrm{cm}^{2}$ ) and thus causing an over-all power conversion efficiency (PCE) of a moderate 2.68\%. Terpolymers P1 and P2, which contain a second weaker acceptor unit as well (BT and TPD, respectively), showed greatly improved short-circuit 5 currents and also significantly higher fill factors (Table 3). P2 moreover afforded a slightly higher $V_{\mathrm{OC}}(0.72 \mathrm{~V}$ versus $0.68 \mathrm{~V}$ for P1) in agreement with the lower HOMO value measured for this polymer (Table 2). Consequently, the PCE of the device with P1 was nearly doubled to $5.14 \%$ while P2 displayed a PCE of ${ }_{10} 4.23 \%$. P3 and P4, where increasing amounts of DPP is substituted with BTT (12.5\% and $25 \%$ respectively), also showed significantly improved OPV device performances relative to the parent BTT-DPP polymer. Especially the $J_{\mathrm{SC}}$-values are greatly improved and nearly doubled for $\mathbf{P 4}\left(12.07 \mathrm{~mA} / \mathrm{cm}^{2}\right)$. Again, in 15 agreement with the measured HOMO energy levels, P4 has a slightly reduced $V_{\mathrm{OC}}$ as compared to $\mathbf{P 3}$. Mainly due to a higher fill factor the P3-containing device, with a power conversion efficiency of $4.58 \%$, performs marginally better than the device with P4 (PCE of $4.28 \%$ ). We note that these encouraging results 20 are achieved without device optimization and without specific thermal annealing steps or the use of additives.

The external quantum efficiencies (EQEs) are displayed in Figure 2 (right) for all five polymer : $\mathrm{PC}_{71} \mathrm{BM}$ devices. The BTTDPP device exhibited a broad response from $350 \mathrm{~nm}$ extending 25 beyond $800 \mathrm{~nm}$, although the efficiency is rather low with a maximum EQE of $25 \%$ at $490 \mathrm{~nm}$. From a comparison with the optical absorption profile of BTT-DPP (Figure 1) it is clear that a large proportion of the electrical charge is generated from direct optical excitation of $\mathrm{PC}_{71} \mathrm{BM}$. This is indicative of a non-optimal 30 energy offset for electron transfer from polymer donor to fullerene acceptor as also reported in our previous study of BTTDPP. ${ }^{13} \mathbf{P 1}$ and $\mathbf{P 2}$ show much higher EQEs with peak values of $41 \%$ and $42 \%$ respectively at $490 \mathrm{~nm}$. The lack of charge photogeneration from polymer excitons is even more pronounced 35 for P2; an observation which correlates well with the lower LUMO level of this material (Table 2) and hence an even smaller energy offset for charge transfer from donor to acceptor. P3 and especially $\mathbf{P 4}$, on the other hand, show improved photogeneration from polymer excitons as evident from the much larger EQE 40 response in the $650-800 \mathrm{~nm}$ region. The improved charge transfer is in good agreement with the slightly raised LUMO energy of P4. EQE values of $37 \%(490 \mathrm{~nm})$ and $24 \%(750 \mathrm{~nm})$ were measured for P3, while EQEs of $43 \%$ at $500 \mathrm{~nm}$ and $33 \%$ at $750 \mathrm{~nm}$ were found for $\mathbf{P} 4$.
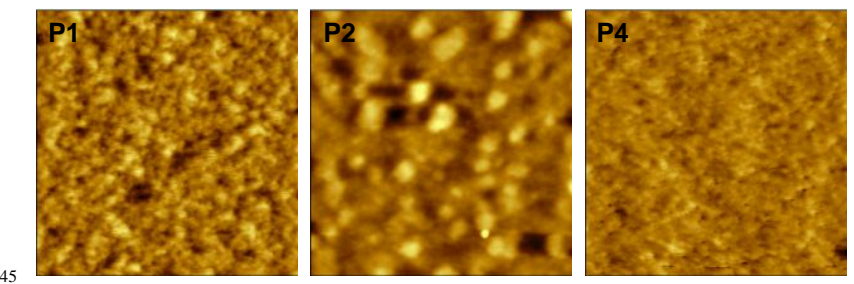

Figure 3 AFM images (tapping-mode, $2 \times 2 \mu \mathrm{m}$ ) of polymer: $\mathrm{PC}_{71} \mathrm{BM}$ (1:2) blends spin-cast from chloroform:o-dichlorobenzene (4:1). See Supporting Information for the BTT-DPP and P3 blend images.

Atomic force microscopy (AFM) was applied to examine the 50 surface morphology of the blend films and the resulting micrographs are depicted in Figure 3. The blend film with BTT-
DPP (see Supporting Information) appears to have the coarsest phase separation, while the domains in the P1-blend seem to be slightly smaller. A much rougher film surface is achieved with $\mathbf{P 2}$ 55 and there is clear evidence of some larger crystalline domains being formed. These crystalline domains seem to be intermixed with a very homogeneous phase and could account for the reduced photocurrent of the device with $\mathbf{P 2}$ relative to the other three random terpolymers. P3 and P4, on the other hand, give 60 very homogeneous films as illustrated for the blend with P4 in Figure 3. All polymer:fullerene blends in this study clearly yield good quality thin films upon solvent casting as evident from the AFM micrographs. Although slight variations in surface morphologies are observed for these films, there are no ${ }_{65}$ indications of highly unfavorable phase segregation for any of the blends. This correlates well with the good OPV performance observed for all the materials discussed herein.

\section{Conclusions}

In conclusion, through a highly modular synthetic approach, we 70 have illustrated how high molecular weight random donoracceptor terpolymers can be utilized to improve polymer solubility and processability, and to broaden the optical absorption and therefore improve the light-harvesting properties. Through variation of the third component in the terpolymers, we 75 have successfully managed to fine tune the frontier energy levels and clearly illustrated the benefits of this approach in relation to OPV device performance. Lowering of the HOMO energy level, as in the case of terpolymer $\mathbf{P 2}$, afforded an improved $V_{\text {oc }}$, while raising the LUMO energy level (P4) improved the charge transfer 80 and consequently more than doubled the EQE in the longwavelength region. All presented terpolymers gave highly efficient OPV devices displaying high currents and high fill factors and the PCE of $5.14 \%$ for the BT-containing terpolymer P1 represents nearly a doubling of the performance of the parent 85 alternating copolymer BTT-DPP.

\section{Notes and references}

${ }^{a}$ Department of Chemistry and Centre for Plastic Electronics, Imperial College London, London, SW7 2AZ, UK, E-mail:

c.nielsen@imperial.ac.uk

${ }_{90}{ }^{b}$ Department of Physics and Centre for Plastic Electronics, Imperial College London, London, SW7 2AZ, UK

${ }^{c}$ CSIRO Materials Science and Engineering, VIC 3169, Australia

${ }^{d}$ Materials Research Center for Information Display, Kyung Hee University, Yongin, Gyeonggi-do 446-701, Korea

$95 \dagger$ Electronic Supplementary Information (ESI) available: Experimental details and characterization data (TGA and DSC), apsorption spectra of the polymer:fullerene blends and additional AFM images. See DOI: $10.1039 / \mathrm{b} 000000 \mathrm{x} /$

1 A. Facchetti, Chem. Mater., 2011, 23, 733.

1002 C. L. Chochos, S. A. Choulis, Prog. Polym. Sci., 2011, 36, 1326.

3 C. J. Brabec, S. Gowrisanker, J. J. M. Halls, D. Laird, S. Jia, S. P. Williams, Adv. Mater., 2010, 22, 3839.

4 J. Kirkpatrick, C. B. Nielsen, W. Zhang, H. Bronstein, R. S. Ashraf, M. Heeney, I. McCulloch, Adv. Energy Mater., 2012, 2, 260.

1055 J. Y. Kim, K. Lee, N. E. Coates, D. Moses, T. Q. Nguyen, M. Dante, A. J. Heeger, Science, 2007, 317, 222.

6 L. Dou, J. You, J. Yang, C.-C. Chen, Y. He, S. Murase, T. Moriarty, K. Emery, G. Li, Y. Yang, Nat. Photonics, 2012, 6, 180.

7 P. M. Beaujuge, S. Ellinger, J. R. Reynolds, Nat. Mater., 2008, 7, 110795 
8 J. Li, K. H. Ong, S. L. Lim, G. M. Ng, H. S. Tan, Z. K. Chen, Chem. Commun., 2011, 47, 9480.

9 P. P. Khlyabich, B. Burkhart, C. F. Ng, B. C. Thompson, Macromolecules, 2011, 44, 5079.

510 C. H. Chen, Y. J. Cheng, C. Y. Chang, C. S. Hsu, Macromolecules, 2011, 44, 8415.

11 C. B. Nielsen, J. M. Fraser, B. C. Schroeder, J. Du, A. J. P. White, W. Zhang, I. McCulloch, Org. Lett., 2011, 13, 2414.

12 C. B. Nielsen, B. C. Schroeder, A. Hadipour, B. P. Rand, S. E. Watkins, I. McCulloch, J. Mater. Chem., 2011, 21, 17642.

13 S. D. Dimitrov, C. B. Nielsen, S. Shoaee, T. P. Shakya, J. Du, I. McCulloch, J. R. Durrant, J. Phys. Chem. Lett., 2012, 3, 140.

14 I. McCulloch, R. S. Ashraf, L. Biniek, H. Bronstein, C. Combe, J. E. Donaghey, D. I. James, C. B. Nielsen, B. C. Schroeder, W. Zhang, Acc. Chem. Res., 2012. DOI: 10.1021/ar200208g. 